\title{
Der Beitrag der Wirtschaftsinformatik zur Früherkennung und Vermeidung von „Black Swans" bei IT-Projekten
}

\author{
DOI 10.1007/s11576-012-0313-7
}

\section{Der Autor}

Prof. Dr. Hans Ulrich Buhl ( $\varangle)$

FIM Kernkompetenzzentrum Finanz\& Informationsmanagement Universität Augsburg Universitätsstraße 12 86159 Augsburg Deutschland hans-ulrich.buhl@wiwi.uni-augsburg. de

Online publiziert: 2012-02-11

This article is also available in English via http://www.springerlink.com and http://www.bise-journal.org: Buhl HU, (2012) The Contribution of Business and Information Systems Engineering to the Early Recognition and Avoidance of "Black Swans" in IT Projects. Bus Inf Syst Eng. doi: 10.1007/ s12599-012-0206-8.

(C) Gabler Verlag 2012
Das Phänomen sogenannter „Black Swans“ erreicht im Kontext von IT-Projekten immer größere Aufmerksamkeit. Auslöser für diese gestiegene Popularität ist unter anderem eine Studie der University of Oxford, aus der hervorgeht, dass es oftmals extrem seltene, aber durchaus schwerwiegende Events sind, die IT-Projekte zum Scheitern bringen (Flyvbjerg und Budzier 2011).

Der ehemalige Investmentbanker Nassim Nicholas Taleb hat den Begriff „Black Swan“ geprägt und beschreibt diesen als höchst unwahrscheinliches Ereignis, welches im Wesentlichen drei Charakteristika aufweist: Unvorhersehbarkeit, massive Auswirkungen und seltenes Auftreten (Taleb 2008). Der Begriff „Black Swan“ geht dabei auf den langjährigen Glauben zurück, dass alle Schwäne weiß seien. Dieser Glauben resultierte ganz einfach aus der Tatsache, dass in Europa ausschließlich weiße Schwäne beobachtet wurden. Die erstmalige Entdeckung eines schwarzen Schwans in Australien kam demnach wie ein Schock, denn sie widersprach allen bisherigen Beobachtungen und somit scheinbar geltenden Gesetzmäßigkeiten. Diese Erkenntnis prägte den metaphorischen Gebrauch des Begriffs „Black Swan“ für ein irrtümlicherweise als unmöglich angesehenes Event.

Insbesondere bei IT-Projekten können „Black Swans“ verheerende Wirkungen haben. So führen sie bspw. dazu, dass jedes sechste IT-Projekt das Budget um ca. 200 \% überschreitet (Flyvbjerg und Budzier 2011). Bei einer genaueren Analyse des Auftretens von „Black Swans“ bei IT-Projekten wurde herausgefunden, dass eine der Hauptursachen im Bereich der Projektplanung begründet liegt (Kendrick 2008). In diesem Bereich sind vor allem Risiken bezüglich der Bewertung von IT-Projekten sowie bezüglich der Berücksichtigung von Abhängigkeiten zu nennen:

- Bewertung von IT-Projekten: Eine der Hauptursachen für das Auftreten von „Black Swans" ist die Tatsache, dass sowohl der Ertrag als auch das Risiko von IT-Projekten primär auf Basis von Erfahrungen von Mitarbeitern geschätzt werden. Dabei zeigen Studien, dass Projektverantwortliche häufig zu optimistische Schätzungen abgeben oder die organisatorische Leistungsfähigkeit falsch einstufen. Da ein Projekt seiner Definition nach ein individuelles Unterfangen darstellt, stellt die adäquate Identifikation sowie die Bewertung der wesentlichen Einflussfaktoren auf das Projekt eine enorme Herausforderung dar. Gerade bei einer steigenden Zahl an IT-Projekten, welche unweigerlich eine erhöhte Komplexität mit sich bringen, können Fehleinschätzungen zu verheerenden Schäden führen. Trotz dieser Herausforderungen finden heute dennoch - wenn überhaupt - oft nur Ex-ante-Bewertungen statt, d. h. einmalige Bewertungen vor Projektbeginn, um eine (open loop) Entscheidung über die Durchführung einer Investition zu treffen, ohne hierbei die späteren (closed loop) Reaktionsmöglichkeiten zu berücksichtigen. Danach erfolgt häufig so lange ein Blindflug, bis größere Probleme nicht mehr zu übersehen und Sanierungsentscheidungen unvermeidbar sind. Dieses Vorgehen vernachlässigt aber den Aspekt, dass sich der Ablauf von IT-Projekten häufig ändert, bspw. aufgrund von neuen Anforderungen. Vor allem langfristige IT-Projekte, welche meist durch ein dynamisches Umfeld maßgeblich beeinflusst werden, können durch einmalige Ex-ante-Bewertungen alleine sicher nicht sinnvoll bewertet werden. Es lässt sich also festhalten, dass einmalige Ex-ante-Bewertungen von IT-Projekten nicht ausreichend sind, um komplexe und dynamische IT-Projekte ganzheitlich bewerten und steuern zu können.

- Abhängigkeiten von IT-Projekten: Die oben angesprochene Komplexität resultiert nicht zuletzt auch aus Abhängigkeiten zwischen IT-Projekten oder anderen ITAssets. So sind bspw. Verzögerungen bei IT-Projekten häufig darauf zurückzuführen, dass Verzögerungen bei vernetzten Projekten auftreten. Gleichzeitig können 
aber auch Abhängigkeiten zu bestehenden IT-Assets, bspw. zu spezieller Software oder Hardware, einen Risikofaktor darstellen, sobald deren Verfügbarkeit kritisch für den Projekterfolg ist. Somit bergen Abhängigkeiten die Gefahr, dass der Erfolg eines IT-Projekts nicht nur durch den eigenen, sondern durch den Erfolg abhängiger IT-Projekte bzw. die Verfügbarkeit von IT-Assets beeinträchtigt wird. Das bedeutet aber auch zugleich, dass ein „Black Swan“ auch Einfluss auf alle abhängigen IT-Projekte haben kann. Somit stellen die Identifikation sowie die Berücksichtigung von Abhängigkeiten bei der Projektplanung einen kritischen Erfolgsfaktor dar.

Um das Ausmaß der „Black Swans“ nun konkreter darzulegen, werden im Folgenden zwei reale IT-Projekte beschrieben, die sich nachträglich als „Black Swans“ herausgestellt haben.

- Das (teils private, teils öffentliche) IT-Projekt „eGK - elektronische Gesundheitskarte" in Deutschland hat zum Ziel, die Abläufe im Gesundheitswesen moderner und wirtschaftlicher zu gestalten. Um dieses Ziel zu erreichen, müssen ca. 110.000 Ärzte, ca. 2.000 Krankenhäuser und ca. 300 Krankenkassen miteinander vernetzt werden. Diese Zahlen verdeutlichen bereits, dass dieses Projekt eine enorme Komplexität aufweist. Diese Komplexität spiegelt sich auch in den veröffentlichten Nutzen-KostenSchätzungen des Projekts wider: Im Jahr 2001 wurden - vermutlich über den Daumen gepeilt - die einmaligen Anfangsinvestitionen auf 1 Milliarde DM = 511 Millionen Euro geschätzt, zusätzlich wurden laufende Auszahlungen von 70 Millionen Euro per annum antizipiert. Der geschätzte Nutzen dagegen belief sich auf lediglich 275 Millionen Euro per annum. Drei Jahre später wurde die Schätzung für die Investitionshöhe auf bis zu 1,4 Milliarden Euro erhöht, die Schätzung für den Nutzen verdoppelte sich fast auf 516 Millionen Euro per annum. Zusätzliche Schätzungen über den erwarteten Amortisationszeitraum des Projekts schwankten zwischen 31 und 108 Monaten. Im Jahr 2006 wurde die Schätzung der Investitionshöhe letztendlich auf ca. 3 Milliarden Euro um den Faktor 6 hochkorrigiert, während sich die Nutzenschätzung noch nicht einmal verdoppelte - mit entsprechenden Folgen für die Wirtschaftlichkeit. Nach allem was man heute weiß wird es dabei nicht bleiben. Einer der wesentlichen Gründe für diese extremen Nachjustierungen auf Kostenund Nutzenseite war und ist die Tatsache, dass sich aufgrund der großen Komplexität sowie der zahlreichen Stakeholder die Anforderungen des Projekts während der Laufzeit häufig änderten. Dieses Beispiel zeigt, dass eine reine Ex-ante-Bewertung von Nutzen und Kosten bei komplexen IT-Investitionen zwar den aktuellen Stand der Erwartungen widerspiegelt, dieser aber völlig unabhängig von den zukünftigen Entwicklungen des Projekts sein kann. Daher ist es beim Management von komplexen IT-Projekten nicht ausreichend, lediglich Ex-ante-Bewertungen durchzuführen und auf deren Eintreten zu vertrauen (vgl. Mertens 2009).

- Die amerikanische Bekleidungsfirma Levi Strauss \& Company beschloss im Jahr 2003, ihre historisch gewachsene IT-Landschaft u. a. durch ein neues ERP-System zu ersetzen. Das Projekt verlief zunächst nach Plan und so konnte die Firma die Inbetriebnahme ihres neuen ERP-Systems für den amerikanischen Geschäftsbereich im Frühjahr 2008 angehen. Die Inbetriebnahme war zuvor bereits als sehr risikoreicher Schritt eingestuft worden. Im Jahresabschlussbericht 2008 berichtet Levi Strauss \& Company davon, dass Großhändler vor der Implementierung des Systems bewusst mit mehr Ware beliefert wurden, um mögliche Probleme bei der Distribution, die im Zuge der Inbetriebnahme auftreten könnten, zu puffern. Aber die Realität übertraf alle Erwartungen: Technische Schwierigkeiten bei der Integration und Anbindung von Altsystemen führten in letztendlicher Konsequenz dazu, dass alle drei amerikanischen Distributionszentren der Firma für eine komplette Woche einen vollständigen Systemausfall erlitten. Dies führte dazu, dass Kundenbestellungen verloren gingen und der Vertrieb zum Erliegen kam. Dieser Ausfall, der auf die Abhängigkeit sowohl zwischen Altsystem und neuem ERP-System, als auch zu den Vertriebssystemen zurückzuführen ist, senkte das Quartalsergebnis um $98 \%$ gegenüber dem Vorjahr, was einem monetären Schaden von 200 Millionen US-Dollar gleichkommt (vgl. Krigsman 2008).

Nach diesen beiden Beispielen drängt sich die Frage auf, warum es trotz zahlreicher Methoden zur Investitionsbewertung zu diesen Entscheidungen kommen konnte bzw. warum solche Events, die erst weit nach Projektbeginn auftreten, nicht vorab angemessen berücksichtigt wurden.

Ein wesentlicher Grund dafür ist in der Anwendung der heutigen Methoden der Entscheidungstheorie zu suchen. Es existiert eine Vielzahl an theoretischen Modellen, die 
darauf ausgerichtet sind, zufällige Ereignisse in der Investitionsentscheidung zu berücksichtigen. Risiko wird demnach meist als zufällige Abweichung von einem erwarteten Wert verstanden. Gerade bei IT-Projekten sind es aber meist nicht zufällige Ereignisse, die ein Projekt zum Scheitern bringen. Vielmehr wird der Verlauf (und damit letztendlich auch der Erfolg) von IT-Projekten durch eine Vielzahl an bewusst oder unbewusst durchgeführten Handlungen bzw. Unterlassungen (nach dem Prinzip Hoffnung zu Unrecht ignorierte Sachverhalte) bzw. getroffene Entscheidungen maßgeblich beeinflusst. Es lässt sich also festhalten, dass die heute in der Investitionsbewertung übliche Beschränkung des Risikos auf zufällige Abweichungen in diesem Kontext nicht ausreichend ist. Erschwerend kommt hinzu, dass gerade bei IT-Projekten eintretende Risiken durch bestehende Abhängigkeiten, die aufgrund der Vernetzung von IT-Projekten oftmals gegeben sind, ein ungeahntes Ausmaß annehmen können. Durch diese Vernetzung kann das Risiko im schlimmsten Fall systemische Dimensionen annehmen und daher zu einem Domino-ähnlichen und somit selbstverstärkenden Effekt führen.

Aus diesem Grund sollten Entscheidungsträger dazu übergehen, die möglichen Ursachen für „Black Swans“ hinreichend genau zu verstehen und zu bewerten, um sowohl ex ante als auch im Projektverlauf alles zu tun, um deren Auftreten zu vermeiden. An dieser Stelle kann und muss die Wirtschaftsinformatik als Schnittstellendisziplin die Brücke zwischen den Anforderungen der Informationstechnologie und der Anwendung wirtschaftswissenschaftlicher Methoden schlagen, um Entscheidungsträger bei Investitionsentscheidungen dieser Art unterstützen zu können. Die Aufgabe der Wirtschaftsinformatik hierbei ist es, über alle Projektphasen hinweg Informationen für eine wertorientierte Entscheidungsunterstützung bereitzustellen. Dabei soll sie eine Hilfestellung bei der Identifikation und Erhebung der relevanten Informationen leisten, Konzepte erstellen, wie diese Informationen aufbereitet werden können und Methoden bereitstellen, wie auf Basis dieser Informationen Handlungsempfehlungen für das Projektmanagement abgeleitet werden können. Ziel dabei ist die Integration der Informationsbeschaffung und deren Verarbeitung in den Projektbewertungs- und Steuerungsprozess. Im Folgenden soll daher dargelegt werden, wie die Wirtschaftsinformatik in den verschiedenen Projektphasen (vor, während, nach) zur Vermeidung von „Black Swans“ beitragen kann:

- Vor dem Beginn eines IT-Projekts liegt der Fokus auf dessen Bewertung und damit auf der Vergleichbarkeit der verschiedenen Einflussfaktoren. Daher wird ein Business Case auf Basis von monetären Größen berechnet. Zur Bewertung der gesamten Kosten eines IT-Projekts gibt es bereits einige Konzepte (wie bspw. „Total Cost of Ownership (TCO)“ oder „Constructive Cost Model (CoCoMo)"). Während man hierbei versucht, die Kostenseite detailliert zu schätzen, finden die erzielbaren Benefits meist viel zu pauschal Beachtung. Das resultiert unter anderem daraus, dass Benefits in der Regel erst nach dem erfolgreichen Abschluss eines Projekts erzielbar und häufig nur schwer prognostizierbar sind. Jedoch spielen auch die Benefits, die den einzelnen Entscheidungen im Projektverlauf zuordenbar sind bei Investitionsentscheidungen eine bedeutende Rolle. Ausgangspunkt einer Kosten und Benefits integrierenden Bewertung jeder dieser Projektentscheidungen ist die Bewertung der entsprechenden Zahlungsströme. Die Höhe der Projekteinzahlungen ergibt sich dabei einerseits aufgrund "harter Benefits", die direkt bzw. indirekt monetär messbar sind (z. B. Personalkosteneinsparungen), und andererseits aufgrund „weicher Benefits“, die nur schwer zuordenbar und monetär bewertbar sind (z. B. Qualitätsverbesserungen). Auch die projektinduzierten Auszahlungen können in direkt bzw. indirekt monetär messbare (z. B. Lizenzkosten) und nur schwer zuordenbare und monetär messbare Auszahlungen (z. B. Kommunikationskosten) untergliedert werden. Da diese Zahlungsströme nicht mit Sicherheit eintreten, sondern durch Entwicklungen während dem Projektverlauf wesentlich beeinflusst werden, sollten deren mögliche Schwankungen, die als Risiko interpretiert werden können, in die Bewertung einfließen. Darüber hinaus sollte analysiert werden, welche Abhängigkeiten zwischen einem IT-Projekt und anderen Projekten bzw. Prozessen im Unternehmen bestehen. Zum Beispiel wird häufig auf dieselben Ressourcen, wie zum Beispiel die ITInfrastruktur oder speziell geschultes Personal, zurückgegriffen, wodurch ein Projekt auch aufgrund von Ressourcenengpässen in eine Schieflage geraten kann. Aus diesem Grund sollten vor Projektbeginn die antizipierten Abhängigkeiten ebenfalls bei der Projektbewertung berücksichtigt werden. Alle hier beschriebenen Einflussfaktoren (Benefits, Kosten, Risiken und Abhängigkeiten) basieren allerdings auf subjektiven Schätzungen von Projektmitarbeitern, was unweigerlich zu Fehleinschätzungen 
führen kann. Auch hier ist es Aufgabe der Wirtschaftsinformatik, Konzepte zu entwerfen, die diese subjektiven Fehlbewertungen mit Hilfe von Debiasing-Maßnahmen minimieren können. Um ein IT-Projekt auf Basis der genannten Einflussfaktoren ex ante bewerten zu können, sollte im Rahmen der Projektbewertung eine theoretisch fundierte Entscheidungsregel herangezogen werden, welche alle oder zumindest die jeweils relevantesten der oben genannten Aspekte beinhaltet. Somit lässt sich festhalten, dass die Wirtschaftsinformatik einen besseren Beitrag liefern kann und muss, um eine integrierte Bewertung von IT-Projekten zu unterstützen.

Ein weiterer positiver Effekt, der mit Hilfe der oben genannten Herangehensweise erreicht werden kann, ist, dass die Komplexität, welche die Bewertung und Steuerung von IT-Projekten mit sich bringt, durch die erhöhte Transparenz, die ein integrierter und standardisierter Bewertungsansatz schafft, verringert werden kann. Zum Beispiel kann dem Nutzer durch die Entwicklung von standardisierten Bewertungstemplates die Projektbewertung und -steuerung erleichtert und damit Konsistenz bei der Projektbewertung sichergestellt werden. Über eine konzeptionelle Erstellung solcher Templates sowie die Definition einer entsprechenden Entscheidungsregel kann die Wirtschaftsinformatik einen signifikanten Beitrag zur Verbesserung der Ex-ante-Projektbewertung leisten.

- Während der Durchführung eines IT-Projekts ist ein kontinuierliches Projektcontrolling mit Closed-loop-Charakter unumgänglich, da aufgrund von internen (z. B. zu optimistische Schätzungen) und externen (z. B. technologische Entwicklung) Einflüssen eine Reallokation von Ressourcen notwendig werden kann. Im Ex-anteBusiness Case erfolgt eine erste Festlegung der Faktoren Funktionalität, Zeit und Budget eines IT-Projekts über Schätzungen, die zum einen durch veränderte Rahmenbedingungen, aber auch durch im Projektablauf getroffene Entscheidungen und Handlungen einem dynamischen Umfeld gegenüberstehen. Es besteht ein Trade-Off zwischen den drei Faktoren, der im Folgenden verdeutlicht wird: Wird der Fokus des Projekts nach Eintreten einer Veränderung, die den Projektverlauf negativ beeinflusst, auf den Faktor Zeit gelegt, d. h. die pünktliche Erreichung des Projektziels rückt in den Mittelpunkt, kann dies nur zu Lasten der Faktoren Budget und/oder Funktionalität erreicht werden. Eine Reallokation der Ressourcen, in diesem Beispiel die Kürzung der Funktionalität oder die Überschreitung des ursprünglich geplanten Budgets, erfordert eine Neubewertung des Business Case, da sich derartige Änderungen negativ auf die realisierbaren Benefits auswirken können. Der Projektleiter benötigt also Informationen darüber, welche Auswirkungen bewusste Änderungen des Projekts mit sich bringen, um korrigierende Maßnahmen einleiten und damit gegensteuern zu können. Durch ein laufendes Projektcontrolling können damit die im Verlauf eines IT-Projekts auftretenden Änderungen der prognostizierten Zahlungsströme möglichst frühzeitig identifiziert werden. Insbesondere vor dem Hintergrund, dass ein Projekt innerhalb komplexer Zusammenhänge und mit vielschichtigen Abhängigkeiten zu anderen Projekten und Prozessen abläuft, sind die Beobachtung von Veränderungen und das daraus resultierende aktive Steuern von großer Bedeutung. Zum Beispiel können, obwohl im betrachteten Projekt alles nach Plan verläuft, durch ein neu in das Portfolio aufgenommenes Projekt Engpässe entstehen. Auch können sich bei unerwarteten Veränderungen bei abhängigen Projekten bisher nicht erahnte Ressourcenengpässe ergeben, weshalb eine kontinuierliche Aktualisierung der in die Projektbewertung eingeflossenen Daten unumgänglich ist. So können auf Basis der neu verfügbaren Informationen im Vorfeld getroffene Entscheidungen nachjustiert werden. Zudem kann ein solches Vorgehen im Zeitverlauf eine immer genauere Bestimmung des Projektwertes ermöglichen, da bei fortschreitender Zeit vermehrt Informationen über die tatsächliche Realisierung vorab geschätzter Einflussfaktoren vorliegen und man bspw. weiß, welche ex ante berücksichtigten Risiken wirklich eingetreten sind und welche nicht. Während das Controlling von anfallenden Auszahlungen in der Regel bereits im Projektmanagement verankert ist, wird insbesondere die Seite des Benefits-Controllings (d. h. welche Einflussfaktoren wirken auf die Erreichung der prognostizierten Benefits eines Projekts) und damit die Einzahlungsseite vernachlässigt. Dies ist jedoch für ein Monitoring und die Steuerung der Benefits-Realisierung im Projektablauf sowie für ein zeitnahes Aufzeigen von Handlungsbedarf wesentlich. Die Wirtschaftsinformatik sollte daher mit der Erarbeitung von Best Practices, die zum Beispiel den Prozess des kontinuierlichen IT-Controllings sowie zugehörige Bewertungs- und Steuerungstemplates beinhalten können, zu einer wertorientierten Ressourcenallokation im Rahmen der laufenden Projektsteuerung beitragen. 
- Nach Abschluss des Projekts ist eine Prüfung, ob die Projektziele erreicht wurden, sinnvoll - schon allein deshalb, um aus Fehlern für die Zukunft zu lernen. Häufig treten die erzielbaren Benefits eines IT-Projekts allerdings erst lange Zeit nach der erfolgreichen Fertigstellung des Projektes auf. Somit müssen unter Umständen auch Ex-post-Maßnahmen ergriffen werden (wie bspw. die Messung der Nutzerakzeptanz oder der direkt erzielten Einzahlungen), um sicherzustellen, dass die prognostizierten Benefits eines IT-Projekts auch tatsächlich realisiert werden. Dieser Aspekt bleibt bis heute in der Praxis weitgehend unberücksichtigt, wodurch Wertpotenziale verschenkt werden. Ein Ex-post-Controlling der Benefits eines IT-Projekts ist daher wesentlich für die Sicherstellung der Zielerreichung eines IT-Projekts und sollte daher in den Bewertungs- und Steuerungsprozess eines IT-Projekts bzw. IT-Projektportfolios integriert werden.

Die bereits mehrfach genannte Verwendung von standardisierten Bewertungsund Steuerungstemplates über den Projektverlauf hinweg birgt das Potenzial, dass eine Dokumentation über die Entwicklungen der einzelnen Einflussparameter erstellt werden kann. Diese Dokumentation könnte zum einen dabei unterstützen, getroffene Entscheidungen nachvollziehbar und damit transparent zu machen, zum anderen könnte diese wertvolle Erfahrungen für andere, vergleichbare Projekte bereitstellen und somit die Basis für ein „organisationales Lernen“ bilden.

Zusammenfassend lässt sich also festhalten, dass die Wirtschaftsinformatik weit mehr zum Erfolg von IT-Projekten beitragen kann und muss, als lediglich starre Methoden der Investitionsbewertung einmalig und ex ante anzuwenden. Vielmehr ist es die Aufgabe der Wirtschaftsinformatik, die Anwendung eines integrierten Ansatzes zur Projektbewertung und -steuerung unter Einbezug von Benefits, Kosten, Risiken und Abhängigkeiten über den gesamten Lebenszyklus eines Projekts hinweg zu ermöglichen. Die eingangs erwähnten Beispiele zeigen jedoch, dass die hier aufgezeigten Maßnahmen in der Praxis noch viel zu wenig Anwendung finden. Es ist daher noch ein weiter Weg, bis aus der Zusammenarbeit zwischen Praxis und Wissenschaft sowohl wissenschaftlich fundierte als auch praktisch evaluierte Methoden bereitstehen, deren Anwendung dazu beiträgt, „Black Swans“ früher zu erkennen und eine Nachsteuerung und Reallokation der Ressourcen vor dem Hintergrund der Unternehmenswertsteigerung anzustoßen. Die hier vorgeschlagenen Maßnahmen sollen dabei noch keine finalen Lösungsvorschläge - wohl aber Denkanstöße für die weitere Forschung - an der Schnittstelle zwischen Theorie und Praxis darstellen. Nur so können Wege und Möglichkeiten geschaffen werden, das Auftreten von „Black Swans“ zukünftig reduzieren, zumindest deren verheerenden Wirkungen eindämmen und damit den Wertbeitrag von IT-Projekten nachhaltig steigern zu können.

\section{Literatur}

Flyvbjerg B, Budzier A (2011) Double whammy - how ICT projects are fooled by randomness and screwed by political intent. http://users.ox.ac.uk/ mast2876/WP_2011_08_15.pdf. Abruf am 2012-01-23

Kendrick T (2008) Avoiding black swans: managing risks using the PERIL database. http://www.failureproofprojects.com/BlackSwan2008.pdf. Abruf am 2011-12-14

Krigsman M (2008) Levi Strauss: SAP rollout 'substantially' hurt quarter. http://www.zdnet. $\mathrm{com} / \mathrm{blog} /$ projectfailures/levi-strauss-sap-rollout-substantially-hurt-quarter/917. Abruf am 2011-12-14

Mertens P (2009) Fehlschläge bei IT-Großprojekten der Öffentlichen Verwaltung. Arbeitspapier, Fachbereich Wirtschaftswissenschaften, Universität Erlangen-Nürnberg

Taleb NN (2008) Der Schwarze Schwan: Die Macht höchst unwahrscheinlicher Ereignisse, 2. Aufl. Carl Hanser, München 